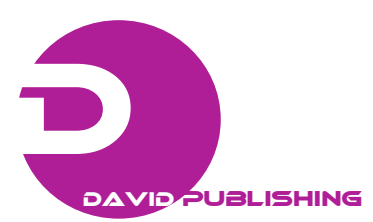

\title{
Design and Manufacture of a Mobile Dynamometer
}

\author{
Animashaun Idriss ${ }^{1}$ and Woods Gerry ${ }^{2}$ \\ School of Mechanical \& Design Engineering, Dublin Institute of Technology, Bolton St, Dublin, D01 K822, Ireland
}

\begin{abstract}
DIT has competed for a number of years in the Formula Student competition with petrol engine propelled vehicles. Dynamometer testing on these engines was traditionally outsourced. In 2017/2018 a decision was made to commence the design of an electric vehicle. Access to a suitable dynamometer for regular testing became more important than ever in order to fully characterise the electric motors the gearbox combinations and optimise the performance of the formula student electric vehicle. This paper deals with the design and manufacture and component selection for a mobile dynamometer that can accurately simulate racetrack conditions and apply typical loading cycles to a motor producing torque, power and speed values from a typical drivetrain. The dynamometer described uses a particle brake to apply the loads, a datum M425 torque transducer to measure torque and a National Instruments LabVIEW interface to display and store data during testing. Safety is of vital importance and this paper describes the high-safety standards applied during the design and manufacture phase. With the implementation of an electronic control circuit, motor characteristics charts are produced, analysed and utilised for calibration and benchmarking for future motor test runs. Aim: Design \& Manufacture of a mobile dynamometer unit to produce torque, speed and power values from the drive train of a motor.
\end{abstract}

Key words: Formula SAE, engine testing, dynamometer, data acquisition, torque transducer.

\section{Introduction}

\subsection{Function}

The function of the engine/motor dynamometer is to impose variable loading conditions on the engine/motor under test across a range of engine speeds and durations. This enables the accurate measurement of the torque and power output from engine/motor [1, 2].

An engine dynamometer was selected for this application, it measures the torque and speed of a drivetrain which in this case is an electric motor. The drive train is directly coupled to the dynamometer during the test phase.

\subsubsection{Absorption Dynamometer}

This is the most common type of dynamometer used for high-accuracy drivetrain testing. They produce a torque by creating constant resistance to a rotating shaft using a PAU (power absorption unit) which achieves this by either mechanical friction,

Corresponding author: Idriss Animashaun, AMEI, B.E in Manufacturing \& Design Engineering, Research \& Development Engineer, Energy Sector. Gerry P Woods, Ph D, Professor, Lecturer \& Chairman of the Manufacturing \& Design Engineering Programme, DIT. fluid friction, or electromagnetic induction. This torque is then measured [1, 2].

There are many aspects to consider when selecting a dynamometer which includes, torque and speed requirements, cost and heat dissipation constraints.

\section{Methodology}

The selected PAU is a magnetic particle brake, Model No. KB-5 from Placid Industries shown in Fig. 1. It was chosen after considering many factors including, the torque produced relative to the size \& weight, and ease of mounting onto drivetrain. It also allows for complete control over the applied torque by adjusting the DC current supplied to the field coil [2].

\subsection{Measurement Instrument}

Instrumentation to measure torque speed and power of the test motor is paramount.

\subsubsection{Torque Transducer M425}

The M425 Torque Transducer seen in Fig. 2 is designed for applications requiring rotary torque measurements. The Transducer will be implemented to fit in line with the dynamometer drivetrain. It 


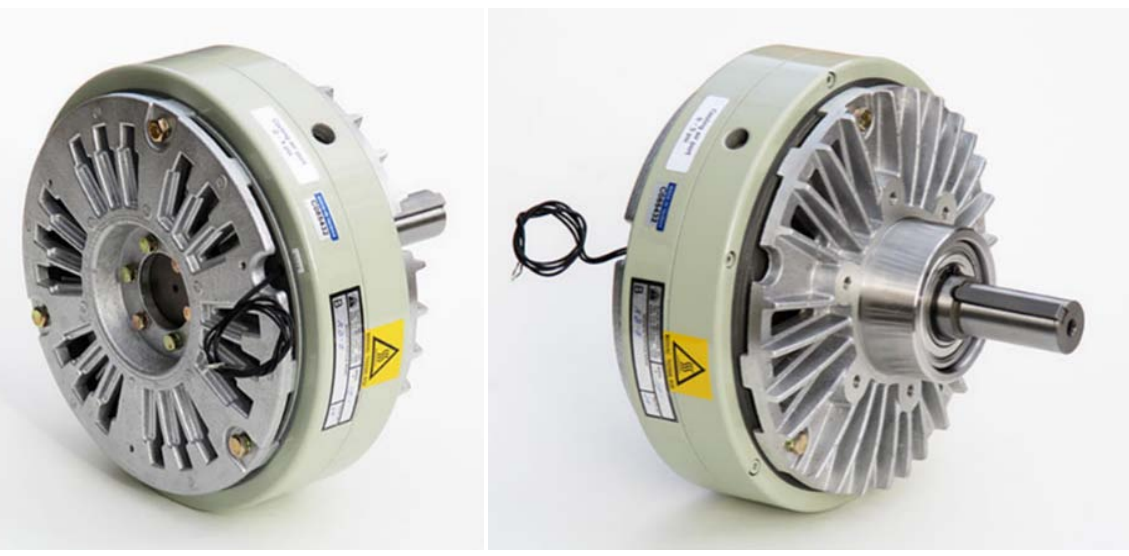

Fig. 1 KB5 placid industries.

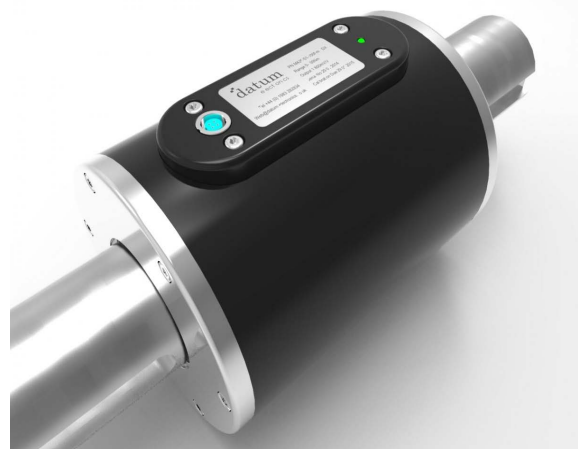

Fig. 2 M425 torque transducer datum electronics.

utilises a non-contact system to transmit data directly proportional to torque. The device also measures the shaft speed and utilises this to calculate power generated [3].

\subsection{Data Acquisition}

The DUI (datum universal interface) is shown in Fig. 3 below. It is designed to work with the M425 torque transducers and guarantees high-accuracy measurement and compatibility. The DUI reads the data from the torque transducer and displays the readings directly onto a built-in display.

A National Instruments Analogue data acquisition unit (NI-DAQ): is shown in Fig. 4 below. It is used to allow the analogue signals for torque, speed and power measured by the DUI to be captured in LabVIEW.

The NI C Series module was selected as it is designed for measurement, control, and communication applications similar to that required in this application.
C Series modules can connect to any sensor or bus and allow for high-accuracy measurements in advanced data acquisition and control applications [4].

\subsection{User Interface}

For the dynamometer to be feasible it must display the most useful data in an easy-to-interpret and intuitive way. This was achieved through the LabVIEW front end shown below in Fig. 5 displaying the essential functions.

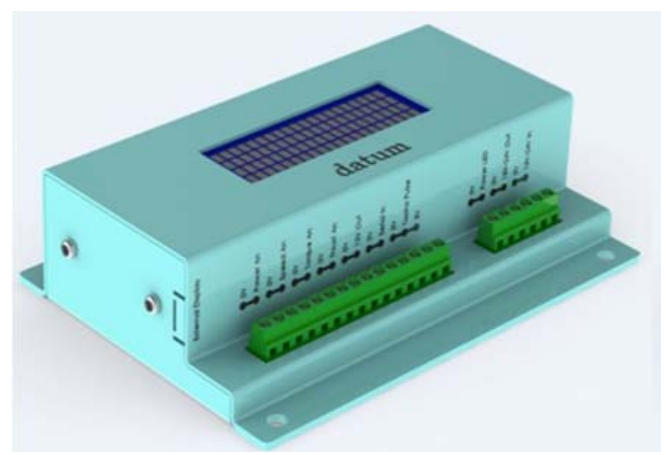

Fig. 3 DUI datum electronics.

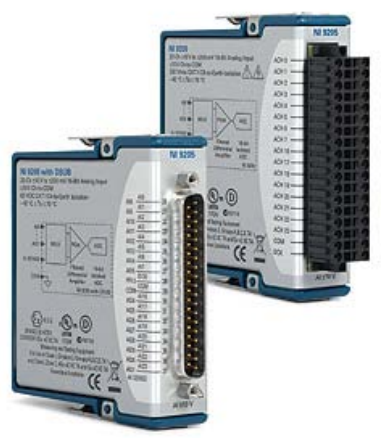

Fig. 4 NI-DAQ National Instruments. 


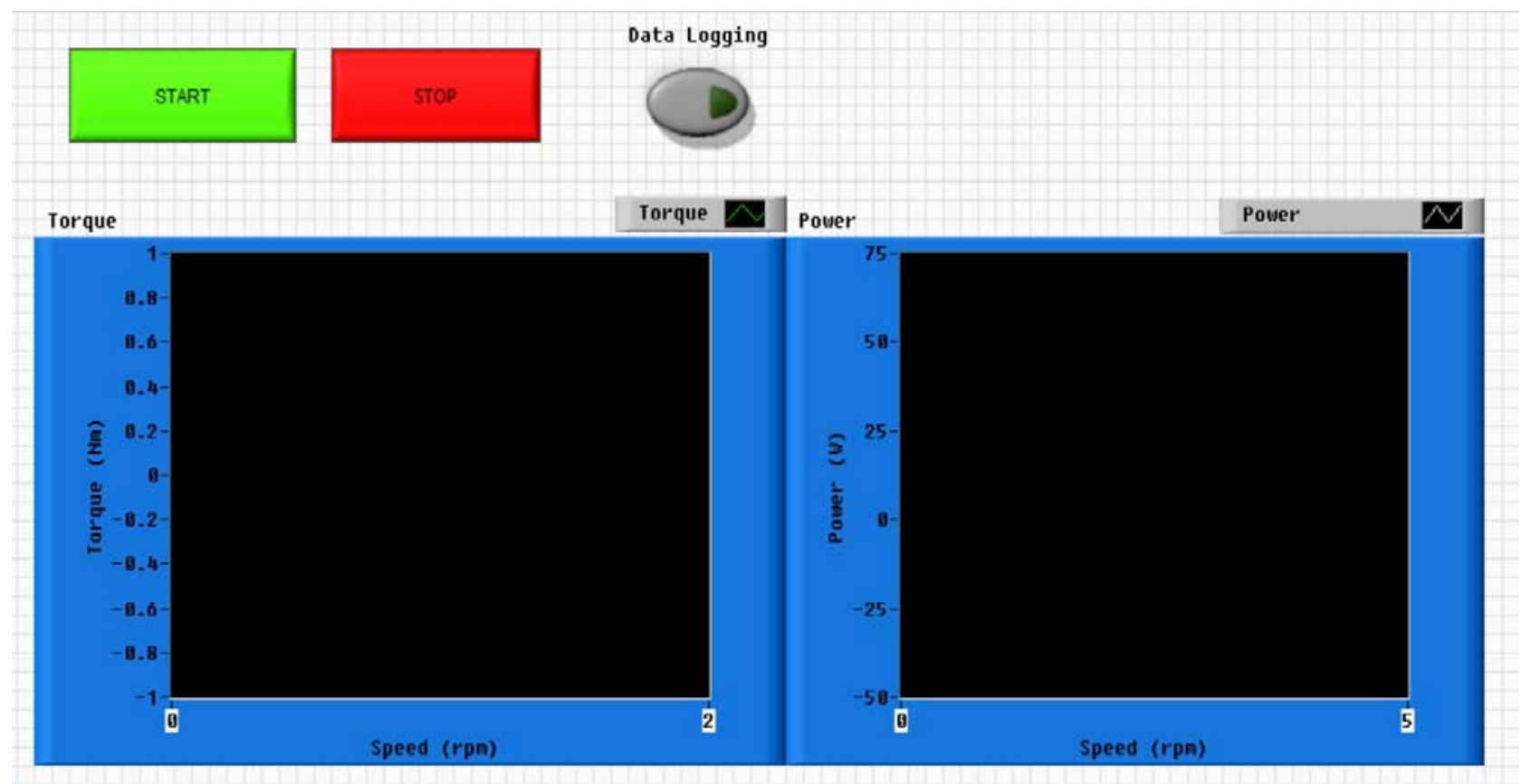

Fig. 5 User interface front panel.

The LabVIEW programme allows the user to start and stop graphing and data logging. The NI MAX program is used to allow LabVIEW to recognise the NI 9205 DAQ hardware and display the data in LabVIEW. The program is explained using a flowchart shown in Fig. 6 [5, 6].

\subsection{Control System}

\subsubsection{Wiring Diagram \& Electrical Components}

A wiring diagram was created to facilitate the ease of assembly of the control system, the wiring diagram shown in Fig. 7 is a visual representation of all the required electrical components for the system and depicts the manner in which they will be wired together. This diagram is used as a reference when selecting electrical components.

\subsubsection{Wiring Diagram Explained}

(A) Power at $380+\mathrm{V}$ is supplied to the unit using a 5-pin plug, the electrical energy first passes through an isolator switch, then a 3-phase circuit breaker.

(B) Next, it passes through a contactor. The emergency stop button and a door interlock are connected inline to the supply which activates the contactor coil.
(C) The three live phases including the neutral and earth cables are connected to a secondary control box which houses a contactor. The coil circuit is made/broken using start and stop push buttons.

(D) One of the live phases is split and passes through a circuit breaker, and powers the DUI, desktop computer and monitor.

(E) It also passes through a power supply unit to convert 220 to $24 \mathrm{~V}$.

(F) This $24 \mathrm{~V}$ circuit goes through a $24 \mathrm{~V}$ redundant circuit safety relay then it passes through a series of switches and interlocks.

(G) $24 \mathrm{~V}$ connects to a latching relay.

(H) The relay sends power to a digital display and connects to a rotary potentiometer that varies the current being supplied to the magnetic particle brake. The supply is read by the digital display.

(I) Another rotary potentiometer is connected to this circuit to reduce the voltage, to supply the NI-9205 with a voltage reading.

(J) Finally, the analogue signals from the DUI are connected to the NI-DAQ and all the analogue signals are sent to the LabVIEW program via serial USB connection [7]. 


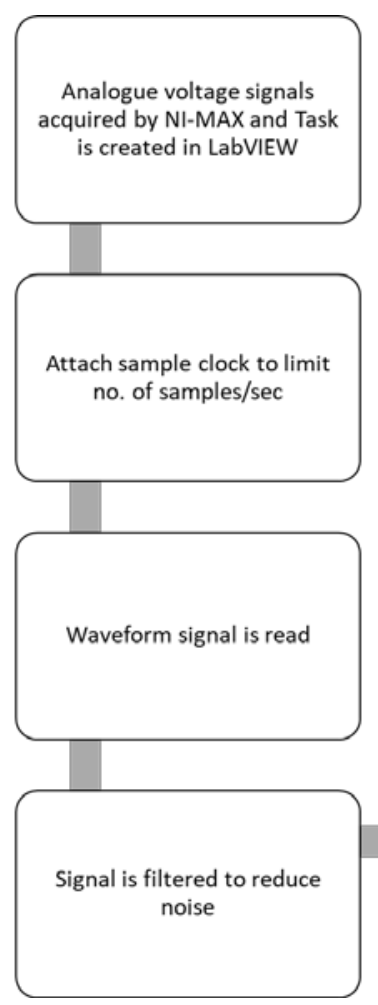

Fig. 6 LabVIEW program.
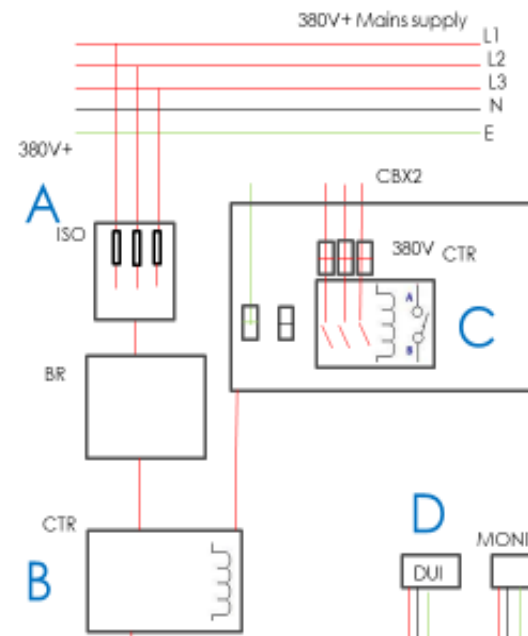

D

BR

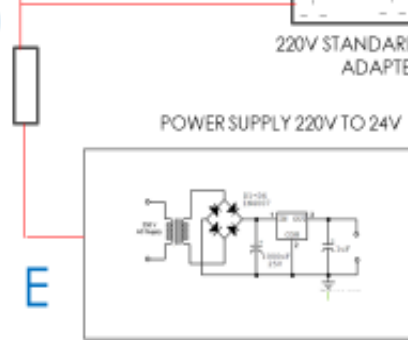

Wiring Diagram Overview

While loop is generated to ensure the program runs continuously
Calibration tests are conducted by comparing characteristic curves produced by the program to those produced by the supplied GUI excel file software

Data points are correlated and used to generate a slope equation to convert the voltage signals to desired units individual graphs
indue are plotted on

Shift register averages last 5 samples to allow for a smooth curve

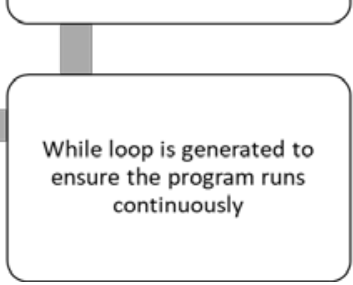

The converted signals are plotted on a individual waveform charts

Voltage signals for Torque, Speed, Power and breaking

torque are converted to desired units (Nm, rpm, W)

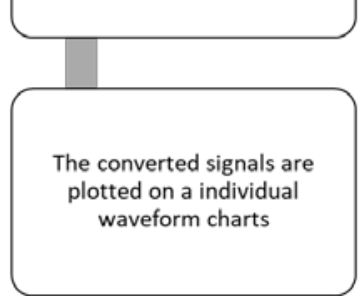




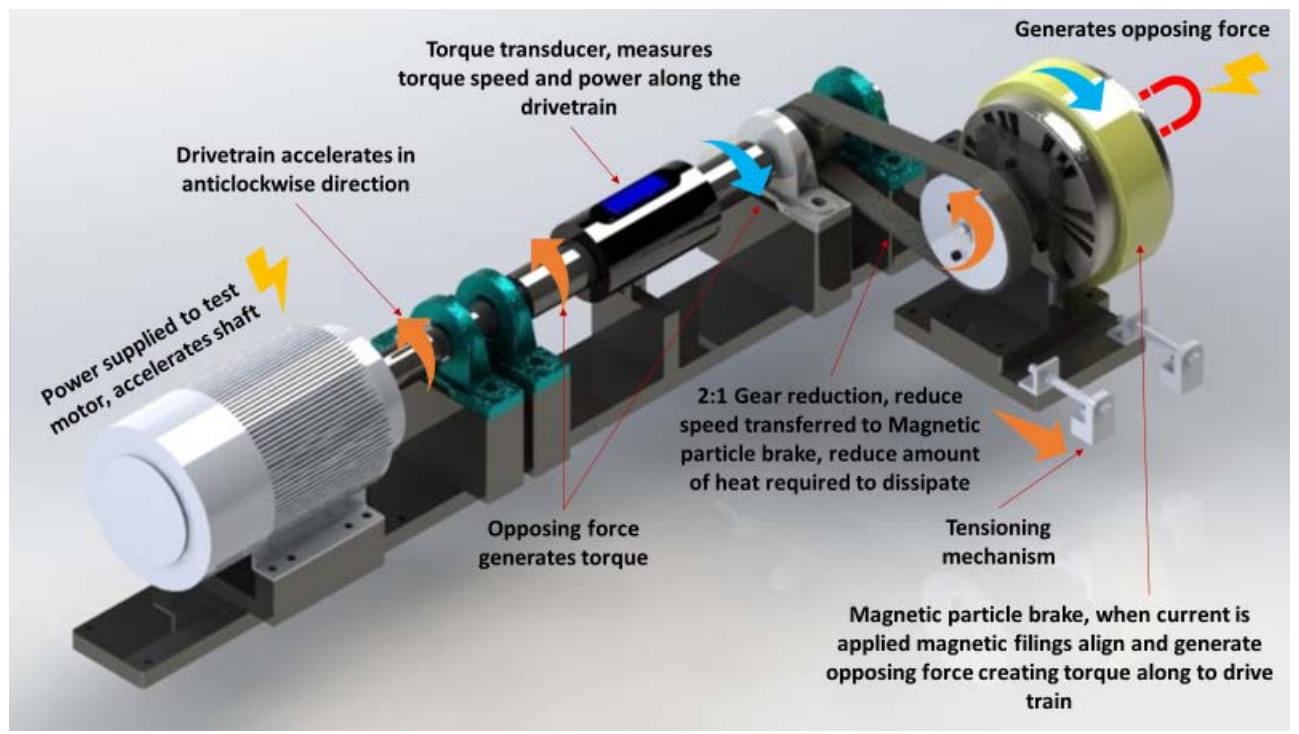

Fig. 8 Drivetrain mechanics.

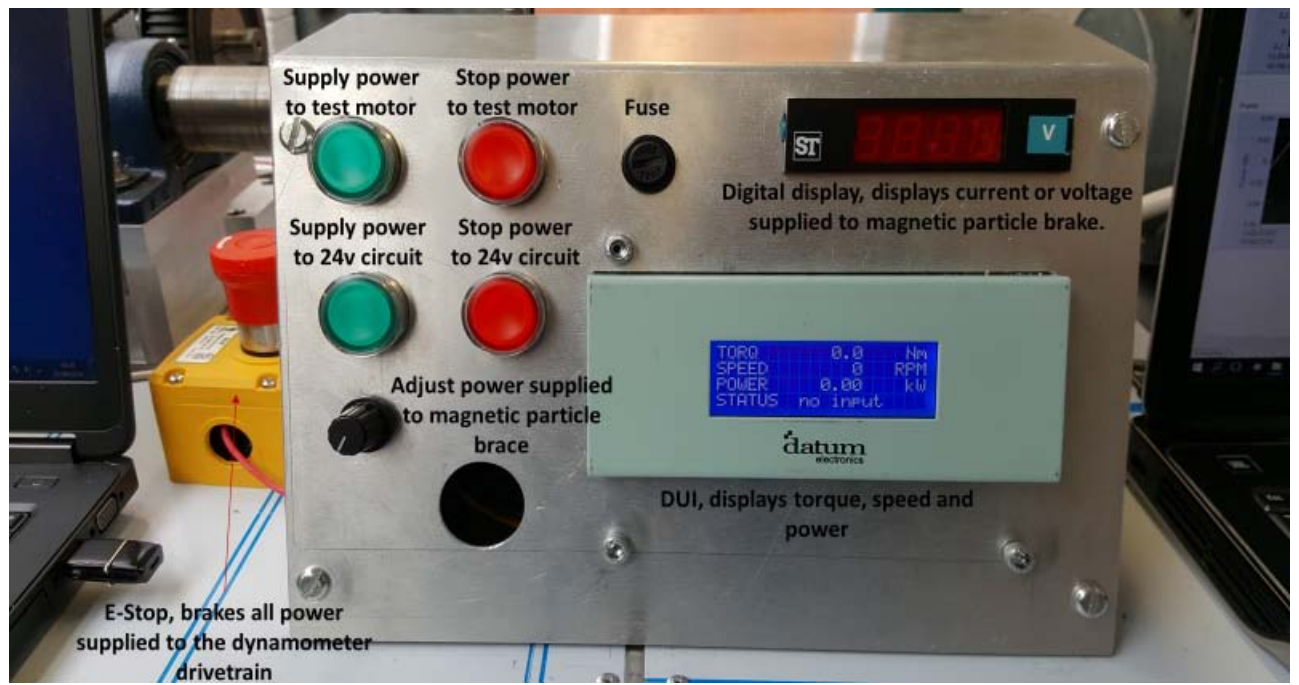

Fig. 9 Control panel.

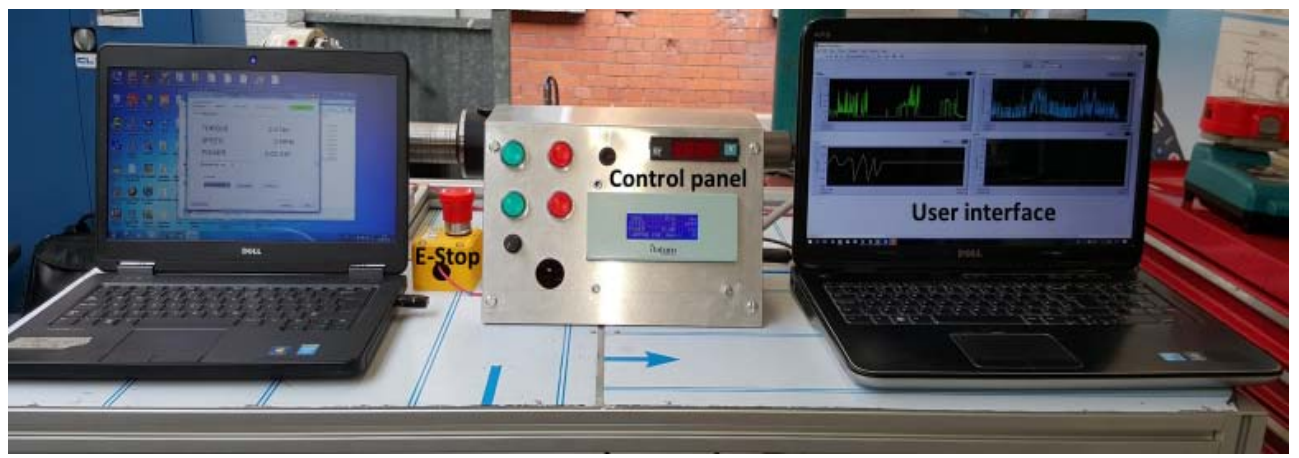

Fig. 10 User interface bed.

Fig. 8 shows a labelled schematic of the dynamometer drivetrain. Power from the mains is supplied to the drivetrain using push buttons mounted on the desktop enclosure shown in Fig. 9. The data acquired from the test run are captured and displayed on a computer screen using the LabVIEW software see Fig. 10. 


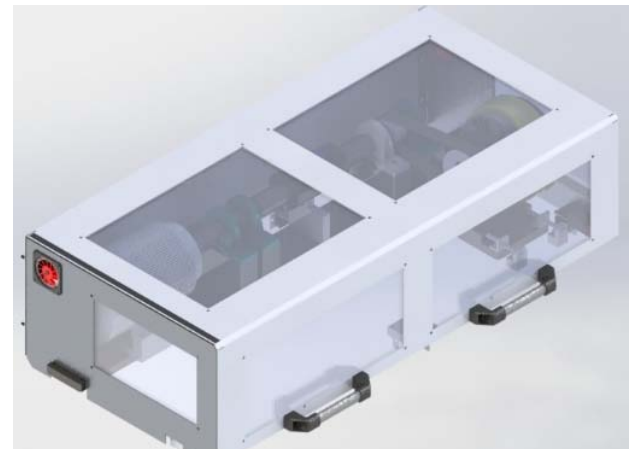

Fig. 11 Guard.

\subsection{Safety Critical Implementation}

Fig. 11 looks at the guarding which covers the entire drive train region, creating a protective interface between the mechanical moving components of the drivetrain and the external environment.

The electrical circuitry incorporated critical safety features such as an emergency stop button, which disconnects all power provided to drivetrain when engaged. A circuit breaker \& Safety relay is employed to protect against excess current running through the system. A safety interlock ensures that the system will not start if the guard \& control box is not locked in the closed position. An isolator is included to shut off power to the entire unit if required. The physical controls contained in the desktop control panel are all wired on the $24 \mathrm{~V}$ circuitry thus, low voltage at the human interface point. This ensures the operator is not exposed to high voltages [7].

\section{Findings}

The characteristic chart produced by the dynamometer is assessed against criteria such as, noise and accuracy. Fig. 12 below shows that as torque is applied to the motor, it draws more current from the power supply to increase power and maintain speed. Electrical noise largely affects the quality of the results. This method of testing can be highly accurate and aid in the selection, and tuning of motors, gearboxes and battery management systems. The main challenge is to reduce the level of electrical noise produced by physical parameters such as, vibration and proximity of wiring. Future expansion of the control system can allow for track simulation and the true value of this method of testing can be assessed.

Electrical noise may be eliminated by determining the natural frequency of the unit to help with vibration management, sensitive electronics can be isolated, and wiring can be arranged in a twisted pair configuration. The final assembly of rig is shown below in Fig. 13.

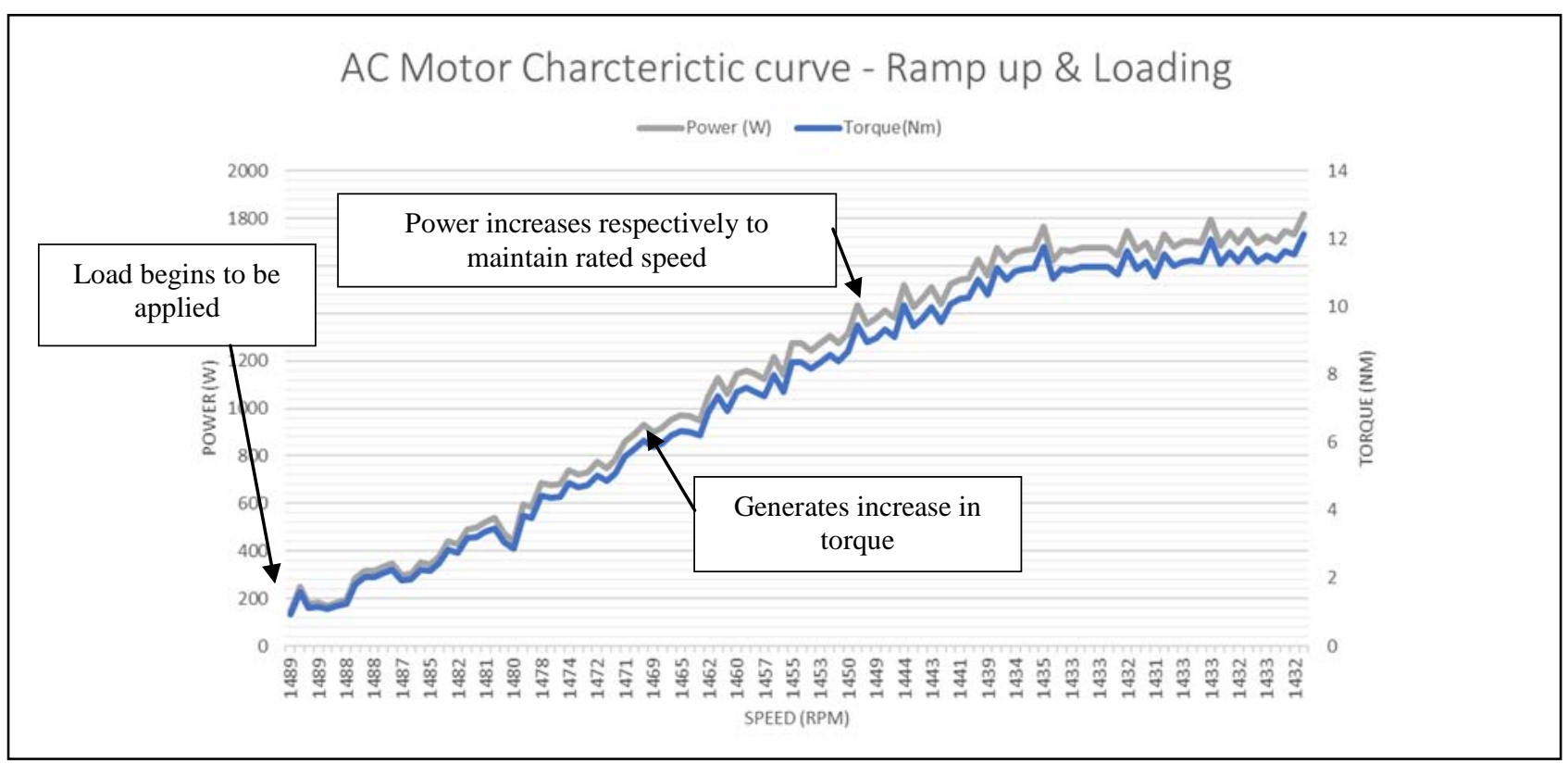

Fig. 12 Characteristic curve. 


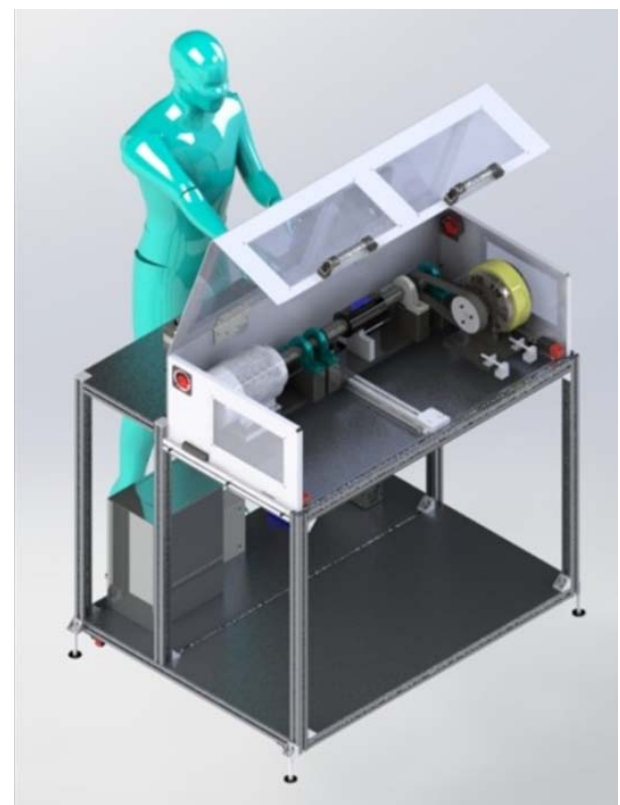

Fig. 13 Dynamometer.

\section{Conclusions}

- Total cost, €5,608.59 (excluding provided components).

- Motor characteristic charts of acceptable quality have been produced.
- Electrical noise affects the quality of the results and further work is needed.

- Current limiting or inverter is required to fully brake Electric motor in a safe manner.

- Particle brake applies braking torque in a smooth and efficient manner, yet heat dissipation is its biggest limiting factor.

\section{References}

[1] Atkins, R. D. 2009. Engine Testing and Development. United States of America: SAE International.

[2] Killedar, J. S. 2012. DYNAMOMETER: Theory and Application to Engine Testing. s.l.: Xlibris, October 25, 2012.

[3] Datum electronics. m425 Torque Transducer Product Overview.

[4] National Instruments. NI 9205 Datasheet.

[5] LabVIEW. Cited: November 1, 2017: http://www.ni.com/en-ie/shop/labview.html.

[6] Kring, J., and T, J. "Introduction to Graphical Programming with LabVIEW. informIT.” Accessed on October 17, 2006. http://www.informit.com/articles/article.aspx?p=662895 \&seqNum=3.

[7] Mercer, B. Industrial control wiring guide. s.l.: Newnes. 